\title{
One Pot Synthesis, Characterisation and Antimicrobial Activity of $\alpha$ - Amino Phosphonates $\uparrow$
}

\author{
V. M. DAYALAN, M. ARTHANAREESWARI, P. KAMARAJ and B. SIVA KUMAR
}

Department of Chemistry, Faculty of Engineering and Technology, SRM University, Kattankulathur-603 203, Tamilnadu, India

dayachem2007@gmail.com

Received 5 February 2013 / Accepted 15 February 2013

\begin{abstract}
The synthesis of some new $\alpha$-amino phosphonates (4a-j) was accomplished by one pot reaction of equimolar quantities of 2-amino-4-chloro-5-nitro phenol (1), aromatic aldehydes (2a-j) and diethyl / dimethylphosphite (3) in dry tetrahydrofuran at reflux condition via Kabachnik - fields reaction in high yields. All the synthesized compounds were characterised by IR, ${ }^{1} \mathrm{H},{ }^{13} \mathrm{C},{ }^{31} \mathrm{P}$ NMR and examined for their antimicrobial activity and found to possess good activity.
\end{abstract}

Keywords: $\alpha$-Amino phosphonates, Kabachnik - fields reaction, Antimicrobial activity

\section{Introduction}

Synthesis of $\alpha$-amino phosphonates exhibiting high bioactivity has recently attracted a lot of attention $^{1-3}$. Applications include inhibition of synthase ${ }^{4}$, HIV protease ${ }^{5}$, renin $^{6}$ and PTPases $^{7,8}$. Some of the derivatives of $\alpha$-amino phosphonates are potential antibiotics ${ }^{9}$ or herbicides ${ }^{10}$, some are chief substrates in the synthesis of phosphoropeptides ${ }^{11}$. Among the number of synthetic approaches to $\alpha$-amino phosphonates, one of the most powerful method is Kabachnik - fields reaction ${ }^{12,13}$. So we made an attempt to synthesise new series of $\alpha$-amino phosphonates.

\section{Experimental}

Chemicals were purchased from Sigma-Aldrich. All of the products were identified by their physical and spectral data. The IR spectra were recorded as KBr pellets on Bruker. ${ }^{1} \mathrm{H},{ }^{13} \mathrm{C},{ }^{31} \mathrm{P}$ NMR were recorded on a Bruker AMX $500 \mathrm{MHz}$ spectrometer operating at $500 \mathrm{MHz}$ for ${ }^{1} \mathrm{H}, 125 \mathrm{MHz}$ for ${ }^{13} \mathrm{C}$ and $202 \mathrm{MHz}$ for ${ }^{31} \mathrm{P}$ NMR. All these compounds were dissolved in DMSO$\mathrm{d}_{6}$. The chemical shifts in $\delta$ were referenced to TMS $\left({ }^{1} \mathrm{H}\right.$ and $\left.{ }^{13} \mathrm{C}\right)$ and $85 \% \mathrm{H}_{3} \mathrm{PO}_{4}\left({ }^{31} \mathrm{P}\right)$.

General procedure for the synthesis of $\alpha$-amino phosphonates (4a-j)

To a stirred solution of 2-amino-4-chloro-5-nitro phenol $(0.003 \mathrm{~mol})$, the aldehyde $(0.003 \mathrm{~mol})$ in anhydrous tetrahydrofuran $(20 \mathrm{~mL})$ was added drop wise and the stirring continued at room temperature for $1 \mathrm{~h}$. Then, dimethyl/ diethyl phosphite $(0.003 \mathrm{~mol})$ in dry

†Presented to the National Conference on Chemistry Solutions at SRM University, India 
tetrahydrofuran $(20 \mathrm{~mL})$ was added drop wise. Stirring was continued at room temperature for another $0.5 \mathrm{~h}$ and the mixture was heated at gentle reflux for $5-6 \mathrm{~h}$. The progress of the reaction was monitored by TLC analysis. After completion of the reaction the solvent was removed under reduced pressure. The residue was purified by column chromatography on silica gel (80-120 mesh) using ethyl acetate: hexane (2:1) as eluent.

\section{Antimicrobial activity}

The antimicrobial activities of the test compounds were evaluated (Table $1 \& 2$ ) and their effect was compared to the stranded antibiotic penicillin and anti-fungal agent griseofulvin. Compounds (4a-j) were screened for their antibacterial activity against Staphylococcus aureus and Escherichia coli by the disc diffusion method ${ }^{18,19}$ in nutrient agar medium at various concentrations $(100,250 \mu \mathrm{g} / \mathrm{disc})$ in dimethyl formamide (DMF). These solutions were added to each filter disc and DMF was used as control. The plates were incubated at $35{ }^{\circ} \mathrm{C}$ and examined for zone of inhibition around each filter disc after $24 \mathrm{~h}$. Their antifungal activity was evaluated against Aspergillus niger and Fusarium oxysporium of 100 and $250 \mu \mathrm{g} /$ disc. Fungal cultures were grown on potato dextrose broth at $25^{\circ} \mathrm{C}$ and finally spore suspension was adjusted to $10^{5}$ spores $/ \mathrm{mL}$. Most of the compounds showed significant activity against bacteria and low activity against fungi.

\section{Results and Discussion}

$\alpha$-Amino phosphonates (4a-j) were synthesized by three component one-pot reaction of equimolar quantities of 2-amino-4-chloro-5-nitro phenol (1), dimethyl/ diethyl phosphite (2) and various aldehydes (3a-j) in dry tetrahydrofuran at reflux conditions for 5-6 h (Scheme 1) . Progress of the reaction was monitored by TLC analysis and products were purified by column chromatography using ethyl acetate:hexane (2:1) as eluent. The chemical structures of all the new compounds were confirmed by elemental analysis, IR ${ }^{1} \mathrm{H},{ }^{13} \mathrm{C}$ and ${ }^{31} \mathrm{P}$ NMR.

Compounds 4a-j exhibited characteristic IR stretching frequencies in the region ${ }^{14}$ 742-769, 1161-1264 and 3314-3404 $\mathrm{cm}^{-1}$ for $\mathrm{P}-\mathrm{C}_{\text {(aliphatic), }} \mathrm{P}=\mathrm{O}$ and $\mathrm{N}-\mathrm{H}$ respectively. The aromatic protons of the two benzene rings of the compounds $4 \mathrm{a}-\mathrm{j}$ showed a mutiplet at $\delta$ 6.42-7.54. The P-C-H proton signal appeared as multiplet ${ }^{15}$ at $\delta 3.92-4.24$ due to its coupling with phosphorus and proton of $\mathrm{N}-\mathrm{H}$. The $\mathrm{N}-\mathrm{H}$ proton signal appeared as singlet. The methylene protons of $\mathrm{P}-\mathrm{OC} \underline{\mathrm{H}}_{2} \mathrm{CH}_{3}$ showed a multiplet and methyl protons of $\mathrm{P}-\mathrm{OCH}_{2} \underline{\mathrm{C}}_{3}$ showed a triplet in the region of $\delta$ 3.55-3.99 and $\delta 1.05-1.38$ respectively ${ }^{16} .{ }^{31} \mathrm{P} N \mathrm{NMR}$ signals ${ }^{17}$ appeared in the region 22.59-27.86 ppm for all the compounds $\mathbf{4 a - j}$.

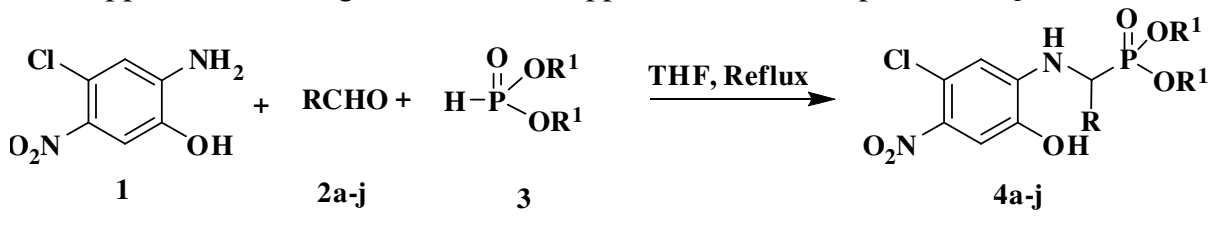

Scheme 1

\begin{tabular}{|c|c|c|c|c|c|}
\hline Compd & $\mathrm{R}$ & $\mathrm{R}^{1}$ & Compd & $\mathrm{R}$ & $\mathrm{R}^{1}$ \\
\hline $2 a \& 4 a$ & $4-\mathrm{OHC}_{6} \mathrm{H}_{4}$ & $\mathrm{C}_{2} \mathrm{H}_{5}$ & $2 f \& 4 f$ & $4-\mathrm{OHC}_{6} \mathrm{H}_{4}$ & $\mathrm{CH}_{3}$ \\
\hline $2 b \& 4 b$ & $3,4-(\mathrm{OMe})_{2} \mathrm{C}_{6} \mathrm{H}_{3}$ & $\mathrm{C}_{2} \mathrm{H}_{5}$ & $2 g \& 4 g$ & $3,4-(\mathrm{OMe})_{2} \mathrm{C}_{6} \mathrm{H}_{3}$ & $\mathrm{CH}_{3}$ \\
\hline $2 c \& 4 c$ & 4- $\mathrm{ClC}_{6} \mathrm{H}_{4}$ & $\mathrm{C}_{2} \mathrm{H}_{5}$ & $2 h \& 4 h$ & 4- $\mathrm{ClC}_{6} \mathrm{H}_{4}$ & $\mathrm{CH}_{3}$ \\
\hline $2 d \& 4 d$ & $2-\mathrm{NO}_{2} \mathrm{C}_{6} \mathrm{H}_{4}$ & $\mathrm{C}_{2} \mathrm{H}_{5}$ & $2 i \& 4 i$ & $2-\mathrm{NO}_{2} \mathrm{C}_{6} \mathrm{H}_{4}$ & $\mathrm{CH}_{3}$ \\
\hline $2 e \& 4 e$ & 4- $\mathrm{OMeC}_{6} \mathrm{H}_{4}$ & $\mathrm{C}_{2} \mathrm{H}_{5}$ & $2 \mathrm{j} \& 4 \mathrm{j}$ & 4- $\mathrm{OMeC}_{6} \mathrm{H}_{4}$ & $\mathrm{CH}_{3}$ \\
\hline
\end{tabular}


Table. 1. Antibacterial activity of $\mathbf{4 a - j}$

\begin{tabular}{|c|c|c|c|c|}
\hline \multicolumn{5}{|c|}{ Zone of inhibition, $\mathrm{mm}$} \\
\hline \multirow{2}{*}{ Compound } & \multicolumn{2}{|c|}{ Escherichia coli } & \multicolumn{2}{|c|}{ Staphylococcus aureus } \\
\hline & $100^{\mathrm{a}} \mu \mathrm{g} / \mathrm{disc}$ & $250^{\mathrm{a}} \mu \mathrm{g} / \mathrm{disc}$ & $100^{\mathrm{a}} \mu \mathrm{g} / \mathrm{disc}$ & $250^{\mathrm{a}} \mu \mathrm{g} / \mathrm{disc}$ \\
\hline $4 \mathbf{4 a}$ & 9 & 16 & 8 & 13 \\
\hline $4 b$ & 7 & 11 & 8 & 14 \\
\hline $4 c$ & 8 & 12 & 6 & 11 \\
\hline 4d & 6 & 10 & - & 9 \\
\hline $4 e$ & - & 10 & 7 & 12 \\
\hline $4 f$ & 8 & 14 & 6 & 12 \\
\hline $4 g$ & 6 & 13 & 5 & 9 \\
\hline $4 \mathrm{~h}$ & 9 & 16 & 9 & 15 \\
\hline $4 \mathbf{i}$ & 5 & 9 & 6 & 10 \\
\hline $4 \mathbf{j}$ & - & 7 & - & - \\
\hline Penicillin $^{\mathrm{b}}$ & & 20 & & 20 \\
\hline
\end{tabular}

${ }^{a}$ Concentration in ppm, ${ }^{b}$ standard reference

Table 2. Antifungal activity of $\mathbf{4 a - j}$

\begin{tabular}{ccccc}
\hline & \multicolumn{4}{c}{ Zone of inhibition, mm } \\
\cline { 2 - 5 } Compound & \multicolumn{3}{c}{ Aspergillus niger } & \multicolumn{2}{c}{ Fusarium oxysporium } \\
\cline { 2 - 5 } & $100^{\mathrm{a}} \mu \mathrm{g} /$ disc & $250^{\mathrm{a}} \mu \mathrm{g} /$ disc & $100^{\mathrm{a}} \mu \mathrm{g} /$ disc & $250^{\mathrm{a}} \mu \mathrm{g} / \mathrm{disc}$ \\
\hline $\mathbf{4 a}$ & 4 & 7 & 3 & 7 \\
$\mathbf{4 b}$ & 5 & 9 & 5 & 8 \\
$\mathbf{4 c}$ & - & 5 & 3 & 5 \\
$\mathbf{4 d}$ & 7 & 9 & - & 5 \\
$\mathbf{4 e}$ & 5 & 8 & 4 & 7 \\
$\mathbf{4 f}$ & 6 & 10 & 6 & 8 \\
$\mathbf{4 g}$ & - & 4 & - & 4 \\
$\mathbf{4 h}$ & - & 6 & 4 & 7 \\
$\mathbf{4 i}$ & 5 & 8 & 5 & 8 \\
$\mathbf{4 j}$ & 3 & 7 & 2 & - \\
Griseofulvin $^{\mathrm{b}}$ & & 21 & & 20 \\
\hline
\end{tabular}

${ }^{a}$ Concentrationin ppm, ${ }^{b}$ standard reference

Physical, analytical, IR and NMR data of compounds 3a-j

[(5-Chloro-2-hydroxy-4-nitrophenylamino)-(4-hydroxyphenyl)methyl] diethyl-phosphonate (4a)

Yield 79\%; m.p. : 172-174 ${ }^{\circ} \mathrm{C}$. IR: $765\left(\mathrm{P}-\mathrm{C}_{(\text {aliphatic })}\right), 1215(\mathrm{P}=\mathrm{O}), 3335(\mathrm{~N}-\mathrm{H}) \mathrm{cm}^{-1} .{ }^{1} \mathrm{H}$ NMR : $\delta 1.15$ (t, $\left.3 \mathrm{H}, \mathrm{P}-\mathrm{OCH}_{2} \mathrm{C}_{3}\right), 1.26\left(\mathrm{t}, 3 \mathrm{H}, \mathrm{P}-\mathrm{OCH}_{2} \underline{\mathrm{C}}_{3}\right), 3.69-3.72\left(\mathrm{~m}, 2 \mathrm{H}, \mathrm{P}-\mathrm{OC}_{2} \underline{\mathrm{CH}}_{3}\right)$, 3.91-3.95 (m, 2H, P-OC $\underline{\mathrm{H}}_{2} \mathrm{CH}_{3}$ ), 4.13-4.18 (m, 1H, P-CH ), 5.41 (s, 1H, N-H), 6.72-7.54 $(\mathrm{m}, 6 \mathrm{H}, \mathrm{Ar}-\mathrm{H}), 10.3(\mathrm{~s}, 1 \mathrm{H}, \mathrm{Ar}-\mathrm{OH}) .{ }^{13} \mathrm{C}$ NMR: $\delta$ 16.7, 55.8, 56.1, 111.2, 113.2, 120.5, 132.5, 141.8, 145.0. ${ }^{31} \mathrm{P}$ NMR: $\delta$ 22.60. Anal.Calcd for $\mathrm{C}_{17} \mathrm{H}_{20} \mathrm{ClN}_{2} \mathrm{O}_{7} \mathrm{P}: \mathrm{C}, 47.38 ; \mathrm{H}, 4.64$; N, 6.50. Found: C, 47.29; H, 4.61; N, 6.45. 
[(5-Chloro-2-hydroxy-4-nitrophenylamino)-(3,4-dimethoxyphenyl)methyl] diethylphosphonate $(\mathbf{4 b})$

Yield 65\% ; m.p. : 189-191 ${ }^{\circ} \mathrm{C}$. IR: 756 (P-C (aliphatic) $), 1196(\mathrm{P}=\mathrm{O}), 3356(\mathrm{~N}-\mathrm{H}) \mathrm{cm}^{-1} .{ }^{1} \mathrm{H}$ NMR : $\delta 1.25\left(\mathrm{t}, 3 \mathrm{H}, \mathrm{P}-\mathrm{OCH}_{2} \mathrm{C}_{3}\right), 1.33\left(\mathrm{t}, 3 \mathrm{H}, \mathrm{P}-\mathrm{OCH}_{2} \underline{\mathrm{C}}_{3}\right), 3.78-3.82\left(\mathrm{~m}, 2 \mathrm{H}, \mathrm{P}-\mathrm{OC}_{2} \underline{\mathrm{H}}_{3}\right)$, 3.95-3.98 (m, 2H, P-OCH$\left.{ }_{2} \mathrm{CH}_{3}\right), 4.19-4.24(\mathrm{~m}, 1 \mathrm{H}, \mathrm{P}-\mathrm{CH}), 5.1(\mathrm{~s}, 1 \mathrm{H}, \mathrm{N}-\mathrm{H}), 6.52-7.34(\mathrm{~m}$, $5 \mathrm{H}, \mathrm{Ar}-\mathrm{H}), 10.1$ (s, $1 \mathrm{H}, \mathrm{Ar}-\mathrm{OH}) .{ }^{13} \mathrm{C} \mathrm{NMR}: \delta 15.4,53.1,56.9,65.0,108.4 .2,115.2,119.5$, 134.5, 139.2, 141.4. ${ }^{31} \mathrm{P}$ NMR: $\delta$ 26.67. Anal.Calcd for $\mathrm{C}_{19} \mathrm{H}_{24} \mathrm{ClN}_{2} \mathrm{O}_{8} \mathrm{P}: \mathrm{C}, 48.05 ; \mathrm{H}, 5.05$; N, 5.90. Found: C, 48.01; H, 5.02; N, 5.85.

[(5-Chloro-2-hydroxy-4-nitrophenylamino)-(4-chorophenyl)methyll]diethyl phosphornate $(4 \mathrm{c})$

Yield:72\% ; m.p.: 161-163 ${ }^{\circ} \mathrm{C}$. IR: 769 (P-C (aliphatic $), 1189(\mathrm{P}=\mathrm{O}), 3346(\mathrm{~N}-\mathrm{H}) \mathrm{cm}^{-1} .{ }^{1} \mathrm{H}$ NMR : $\delta 1.05$ (t, $\left.3 \mathrm{H}, \mathrm{P}-\mathrm{OCH}_{2} \underline{\mathrm{C}}_{3}\right), 1.32\left(\mathrm{t}, 3 \mathrm{H}, \mathrm{P}-\mathrm{OCH}_{2} \underline{\mathrm{C}}_{3}\right), 3.59-3.63\left(\mathrm{~m}, 2 \mathrm{H}, \mathrm{P}-\mathrm{OC}_{2} \underline{\mathrm{H}}_{2} \mathrm{CH}_{3}\right.$ ), 3.82-3.85 (m, 2H, P-OC $\left.\underline{\mathrm{H}}_{2} \mathrm{CH}_{3}\right), 4.09-4.13(\mathrm{~m}, 1 \mathrm{H}, \mathrm{P}-\mathrm{CH}), 5.23(\mathrm{~s}, 1 \mathrm{H}, \mathrm{N}-\mathrm{H}), 6.62-7.52$ (m, $6 \mathrm{H}, \mathrm{Ar}-\mathrm{H}), 10.23$ (s, $1 \mathrm{H}, \mathrm{Ar}-\mathrm{OH}) .{ }^{31} \mathrm{P}$ NMR: $\delta$ 22.59. Anal.Calcd for $\mathrm{C}_{17} \mathrm{H}_{19} \mathrm{Cl}_{2} \mathrm{~N}_{2} \mathrm{O}_{6} \mathrm{P}: \mathrm{C}$, 45.43; H, 4.23; N, 6.23. Found: C, 45.32; H, 4.18; N, 6.19.

[(5-Chloro-2-hydroxy-4-nitrophenylamino)-(2-nitrophenyl)methyl]diethylphosphornate (4d)

Yield: 64\% ; m.p. : $165-167{ }^{\circ} \mathrm{C}$. IR : 747 (P-C (aliphatic) $), 1161(\mathrm{P}=\mathrm{O}), 3399(\mathrm{~N}-\mathrm{H}) \mathrm{cm}^{-1} .{ }^{1} \mathrm{H}$ NMR : $\delta 1.22\left(\mathrm{t}, 3 \mathrm{H}, \mathrm{P}-\mathrm{OCH}_{2} \mathrm{CH}_{3}\right), 1.32\left(\mathrm{t}, 3 \mathrm{H}, \mathrm{P}-\mathrm{OCH}_{2} \mathrm{CH}_{3}\right), 3.65-3.69\left(\mathrm{~m}, 2 \mathrm{H}, \mathrm{P}-\mathrm{OCH}_{2} \mathrm{CH}_{3}\right)$, 3.77-3.81 (m, 2H, P-OC $\left.\underline{H}_{2} \mathrm{CH}_{3}\right), 4.11-4.15(\mathrm{~m}, 1 \mathrm{H}, \mathrm{P}-\mathrm{CH}), 5.22(\mathrm{~s}, 1 \mathrm{H}, \mathrm{N}-\mathrm{H}), 6.52-7.51(\mathrm{~m}$, $6 \mathrm{H}, \mathrm{Ar}-\mathrm{H}), 10.11$ (s, $1 \mathrm{H}, \mathrm{Ar}-\mathrm{OH}) .{ }^{31} \mathrm{P}$ NMR: $\delta$ 24.12. Anal.Calcd for $\mathrm{C}_{17} \mathrm{H}_{19} \mathrm{ClN}_{3} \mathrm{O}_{8} \mathrm{P}: \mathrm{C}$, 44.39; H, 4.13; N, 9.14. Found: C, 44.32; H, 4.08; N, 9.09.

[(5-Chloro-2-hydroxy-4-nitrophenylamino)-(4-methoxyphenyl)methyl] diethylphosphonate (4e)

Yield 76\%; m.p.: 182-184 ${ }^{\circ} \mathrm{C}$. IR : $752\left(\mathrm{P}-\mathrm{C}_{(\text {aliphatic }}\right), 1196(\mathrm{P}=\mathrm{O}), 3348(\mathrm{~N}-\mathrm{H}) \mathrm{cm}^{-1} .{ }^{1} \mathrm{H}$ NMR : $\delta 1.15\left(\mathrm{t}, 3 \mathrm{H}, \mathrm{P}-\mathrm{OCH}_{2} \underline{\mathrm{C}}_{3}\right), 1.28\left(\mathrm{t}, 3 \mathrm{H}, \mathrm{P}-\mathrm{OCH}_{2} \mathrm{C}_{3}\right), 3.53-3.57\left(\mathrm{~m}, 2 \mathrm{H}, \mathrm{P}-\mathrm{OC}_{2} \underline{\mathrm{CH}}_{3}\right)$, 3.75-81 (m, 2H, P-OCH $\left.{ }_{2} \mathrm{CH}_{3}\right), 4.15-21(\mathrm{~m}, 1 \mathrm{H}, \mathrm{P}-\mathrm{CH}), 5.14(\mathrm{~s}, 1 \mathrm{H}, \mathrm{N}-\mathrm{H}), 6.42-7.24(\mathrm{~m}$, $6 \mathrm{H}, \mathrm{Ar}-\mathrm{H}), 10.24$ (s, $1 \mathrm{H}, \mathrm{Ar}-\mathrm{OH}) .{ }^{31} \mathrm{P}$ NMR: $\delta$ 25.67. Anal.Calcd for $\mathrm{C}_{18} \mathrm{H}_{22} \mathrm{ClN}_{2} \mathrm{O}_{7} \mathrm{P}: \mathrm{C}$, 48.59; H, 4.94; N, 6.29. Found: C, 48.52; H, 4.89; N, 6.23.

[(5-Chloro-2-hydroxy-4-nitrophenylamino)-(4-hydroxyphenyl)methyl]dimethyl-phosphonate $(\mathbf{4 f})$

Yield 66\%; m.p. : 186-188 ${ }^{\circ} \mathrm{C} . \mathrm{IR}: 745\left(\mathrm{P}-\mathrm{C}_{(\text {aliphatic }}\right), 1216(\mathrm{P}=\mathrm{O}), 3382(\mathrm{~N}-\mathrm{H}) \mathrm{cm}^{-1} .{ }^{1} \mathrm{H}$ NMR: $\delta 3.43\left(\mathrm{~d}, 3 \mathrm{H}, \mathrm{P}-\mathrm{O}-\underline{\mathrm{C}}_{3}\right), 3,52$ (d, 3H, P-O-C $\left.\underline{\mathrm{H}}_{3}\right), 4.01-4.06(\mathrm{~m}, 1 \mathrm{H}, \mathrm{P}-\mathrm{CH}), 5.1(\mathrm{~s}, 1 \mathrm{H}, \mathrm{N}-$ $\mathrm{H}), 6.56-7.44(\mathrm{~m}, 6 \mathrm{H}, \mathrm{Ar}-\mathrm{H}), 10.2(\mathrm{~s}, 1 \mathrm{H}, \mathrm{Ar}-\mathrm{OH}) .{ }^{31} \mathrm{P}$ NMR: $\delta$ 25.08. Anal.Calcd for $\mathrm{C}_{15} \mathrm{H}_{16} \mathrm{ClN}_{2} \mathrm{O}_{7} \mathrm{P}: \mathrm{C}, 44.72 ; \mathrm{H}, 3.97 ; \mathrm{N}, 6.95$. Found: C, 44.66; H, 3.92; N, 6.91.

[(5-Chloro-2-hydroxy-4-nitrophenylamino)-(3,4-dimethoxyphenyl)methyl] dimethylphosphonate $\mathbf{( 4 g )}$

Yield 74\%; m.p. : 146-148 ${ }^{\circ} \mathrm{C}$. IR: 751 (P-C (aliphatic) $), 1247(\mathrm{P}=\mathrm{O}), 3404(\mathrm{~N}-\mathrm{H}) \mathrm{cm}^{-1} .{ }^{1} \mathrm{H}$ NMR: $\delta$ 3.61 (d, 3H, P-O-C $\left.\underline{H}_{3}\right), 3.72$ (d, 3H, P-O-C $\left.\underline{H}_{3}\right), 3.95-4.01$ (m, 1H, P-CH), 4.92 (s, 1H, N-H), 6.55-7.34 (m, 5H, Ar-OH), $10.26(\mathrm{~s}, 1 \mathrm{H}, \mathrm{Ar}-\mathrm{H}) .{ }^{31} \mathrm{P}$ NMR: $\delta$ 27.23. Anal.Calcd for $\mathrm{C}_{17} \mathrm{H}_{20} \mathrm{ClN}_{2} \mathrm{O}_{8} \mathrm{P}: \mathrm{C}, 45.68 ; \mathrm{H}, 4.47 ; \mathrm{N}, 6.27$. Found: C, 45.61; H, 4.42; N, 6.23. 
[(5-Chloro-2-hydroxy-4-nitrophenylamino)-(4-chorophenyl)methyl] dimethylphosphonate $(\mathbf{4 h})$

Yield 63\% ; m.p. : $195-197{ }^{\circ} \mathrm{C}$. IR: $758\left(\mathrm{P}_{-} \mathrm{C}_{\text {(aliphatic) }}\right), 1198(\mathrm{P}=\mathrm{O}), 3372(\mathrm{~N}-\mathrm{H}) \mathrm{cm}^{-1} .{ }^{1} \mathrm{H}$ NMR: $\delta 3.26$ (d, 3H, P-O-C $\left.\underline{H}_{3}\right), 3.45$ (d, 3H, P-O-C $\left.\underline{H}_{3}\right), 3.90-3.96$ (m, 1H, P-CH), 5.14 (s, 1H, N$\mathrm{H})$, 6.70-7.48 (m, 6H, Ar-H), $10.13(\mathrm{~s}, 1 \mathrm{H}, \mathrm{Ar}-\mathrm{OH}) .{ }^{31} \mathrm{P}$ NMR: $\delta$ 27.36. Anal.Calcd for $\mathrm{C}_{15} \mathrm{H}_{15} \mathrm{Cl}_{2} \mathrm{~N}_{2} \mathrm{O}_{6} \mathrm{P}: \mathrm{C}, 42.75 ; \mathrm{H}, 3.56 ; \mathrm{N}, 6.65$. Found: $\mathrm{C}, 42.68 ; \mathrm{H}, 3.52 ; \mathrm{N}, 6.59$.

[(5-Chloro-2-hydroxy-4-nitrophenylamino)-(2-nitrophenyl)methyl]dimethylphosphonate (4i)

Yield: 70\%; m.p. : $178-180^{\circ} \mathrm{C}$. IR: 751(P-C $($ aliphatic) $), 1264(\mathrm{P}=\mathrm{O}), 3359(\mathrm{~N}-\mathrm{H}) \mathrm{cm}^{-1} .{ }^{1} \mathrm{H}$ NMR: $\delta 3.33\left(\mathrm{~d}, 3 \mathrm{H}, \mathrm{P}-\mathrm{O}-\mathrm{C}_{3}\right), 3.51\left(\mathrm{~d}, 3 \mathrm{H}, \mathrm{P}-\mathrm{O}-\mathrm{C}_{3}\right), 4.13-4.17(\mathrm{~m}, 1 \mathrm{H}, \mathrm{P}-\mathrm{CH}), 5.11(\mathrm{~s}, 1 \mathrm{H}, \mathrm{N}-$ $\mathrm{H}), 6.45-7.54(\mathrm{~m}, 6 \mathrm{H}, \mathrm{Ar}-\mathrm{H}), 10.2(\mathrm{~s}, 1 \mathrm{H}, \mathrm{Ar}-\mathrm{OH}) .{ }^{31} \mathrm{P}$ NMR: $\delta$ 26.78. Anal.Calcd for $\mathrm{C}_{15} \mathrm{H}_{15} \mathrm{ClN}_{3} \mathrm{O}_{8} \mathrm{P}: \mathrm{C}, 41.71 ; \mathrm{H}, 3.47 ; \mathrm{N}, 9.73$. Found: C, 41.64; H, 3.40; N, 9.68.

[(5-Chloro-2-hydroxy-4-nitrophenylamino)-(4-methoxyphenyl)methyl]dimethylphosphonate $(\mathbf{4 j})$

Yield 76\% ; m.p. : 156-158 ${ }^{\circ} \mathrm{C}$. IR: 743(P-C $($ aliphatic $), 1191(\mathrm{P}=\mathrm{O}), 3314(\mathrm{~N}-\mathrm{H}) \mathrm{cm}^{-1} .{ }^{1} \mathrm{H}$ NMR: $\delta 3.65\left(\mathrm{~d}, 3 \mathrm{H}, \mathrm{P}-\mathrm{O}-\mathrm{C} \underline{\mathrm{H}}_{3}\right), 3.79\left(\mathrm{~d}, 3 \mathrm{H}, \mathrm{P}-\mathrm{O}-\mathrm{CH}_{3}\right), 4.17-4.22(\mathrm{~m}, 1 \mathrm{H}, \mathrm{P}-\mathrm{CH}), 5.14(\mathrm{~s}, 1 \mathrm{H}, \mathrm{N}-$ $\mathrm{H}), 6.62-7.34(\mathrm{~m}, 6 \mathrm{H}, \mathrm{Ar}-\mathrm{H}), 10.05(\mathrm{~s}, 1 \mathrm{H}, \overline{\mathrm{Ar}}-\mathrm{OH}) .{ }^{31} \mathrm{P}$ NMR : $\delta$ 27.86. Anal.Calcd for $\mathrm{C}_{16} \mathrm{H}_{18} \mathrm{ClN}_{2} \mathrm{O}_{7} \mathrm{P}: \mathrm{C}, 46.09 ; \mathrm{H}, 4.32 ; \mathrm{N}, 6.72$. Found: C, 46.01; H, 4.27; N, 6.67.

\section{Conclusion}

We have synthesized new $\alpha$-amino phosphonates $\mathbf{4 a - j}$ in high yields by Kabachnik - fields reaction without using any catalyst. All of them showed significant activity against bacteria and low activity against fungi.

\section{Acknowledgement}

The authors express thanks to ISISM, SRM University for providing spectral data and Faridha Begham for her help in the Antimicrobial assay.

\section{References}

1. Kaboudin B and Nazari R, Tetrahedron Lett., 2001, 42, 8211.

2. Matveeva E D, Podrugina T A, Tishkovskaya E V, Tomilova L G and Zefirov N S, Synlett., 2003, 2321-2324.

3. Pavlov V Ya, Kabachinik M M, Zobnina E V, Ponomarev E V and Beletskaya I P, Synlett., 2003.

4. Sikorski J A, Miller M J, Braccolino D S, Cleary D G, Corey S D, Ream J E, Schnur D, Shah A and Walker M C, Phosphorus Sulfur Silicon, 1993, 76, 375.

5. Stowasser B, Budt K H, Jain-Qi L, Peyman A and Ruppert D, Tetrahedron Lett., 1992, 33, 6625.

6. Patel D V, Reilly-Gauvin K and Ryono D E, Tetrahedron Lett., 1990, 31, 5587-5590;

7. (a) Bruke T R, Jr Brachi J J, Jr George C, Wolf G, Shoelson S E and Yan X, J Med Chem., 1995, 38, 1386; (b) Bruke T R, Jr Kole H K and Roller P P, Biochem Biophys Res Commun., 1994, 204(1), 129-134.

8. Peyman A, Budt K H, Paning J S, Stowasser B and Ruppert D, Tetrahedron Lett., $1992,33,4549$.

9. Atherton F R, Hall M J, Hassall C H and Lambert R W, J Med Chem., 1986, 29(1), 29-40. 
10. Kafarski P, Lejack B and Mastalerz P, Beitr Wirk Forsh., 1985, H25; Chem Abstr., 1985, 103, 174532.

11. (a) Maier L and Lea P J, Phosphorus Sulphur Silicon, 1983, 17, 1; (b) Giannousis P P and Bartlett P A, J Med Chem., 1987, 30, 1603; (c) Gancarz R and Wieczorek J S, Synthesis, 1977, 625; (d) Baylis E K, Campbell C D and Dingwall J G, J Chem Soc Perkin Trans., 1984, 1, 2845.

12. (a) Kabachnic M J and Medved T, Izv Akad Nauk SSSR, 1953, 1126; (b) Kabachnic M J and Medved T, Izv Akad Nauk SSSR, 1954, 1024.

13. Fields E K, J Am Chem Soc., 1952, 74(6), 1528-1531.

14. Silverstein R M, Bassler G C and Morill T C, Spectrometric Identification of Organic Comounds, $6^{\text {th }}$ Ed., John Wiley \& Sons: New York, 1991.

15. Tongcharoensirikul P, Suarez A I, Volker T and Thomson C M, J Org Chem., 2004, 69, 2322-2326.

16. Pettersen D, Marcolini M, Bernardi L, Fini F, Herrera R P, Sgarzani V and Ricci A, $J$ Org Chem., 2006, 71(16), 6269-6272.

17. Quin L D and Verkade J G, Phosphorus-31 NMR Spectral Properties in Compound Characterization and Structural Analysis, VCH P: New and Structural Analysis, VCH P: New York, 1994.

18. Cruickshan R, Medical Microbiology, A guide to diagnosis and control of infection, II, Ed., Livingston E S, Ltd: Edinburgh and London, 1968.

19. Beuer A W, Kirby M M, Sherries J C and Truck A, Am J Clin Pathol., 1969, 45, 493-496. 